\title{
Cómo diagnosticamos y tratamos una enfermedad reumática: casos clínicos interactivos
}

\author{
E. Andreu Alapont ${ }^{\mathrm{a}}$, L. Lacruz Pérez ${ }^{\mathrm{b}}$, B. López Montesinos ${ }^{\mathrm{c}}$, I. Calvo Penadés ${ }^{\mathrm{d}}$ \\ apediatra. CS Quart de Poblet. Valencia. España. \\ bPediatra. Unidad de Reumatología. Hospital Universitario Son Dureta. Palma de Mallorca. España. \\ Unidad de Reumatología. Hospital Universitario La Fe. Valencia. España. \\ dPediatra. Unidad de Reumatología Pediátrica. Hospital Infantil La Fe. Valencia. España.
}

\begin{abstract}
Resumen
En este seminario se trata el abordaje diagnóstico y terapéutico inicial de las enfermedades reumáticas pediátricas a través de la resolución interactiva de unos casos clínicos representativos de la artritis idiopática juvenil en sus distintas formas de expresión clínica, por ser esta la enfermedad más frecuente en esta especialidad. Se repasan los últimos avances en el diagnóstico y tratamiento de interés para el pediatra de Atención Primaria.

Palabras clave: Artritis idiopática juvenil. Diagnóstico. Tratamiento.
\end{abstract}

How do we diagnose and treat a pediatric rheumatic disease: interactive cases report

Abstract

This seminar approaches the initial diagnosis and treatment of juvenile idiopathic arthritis, the most frequent rheumatic disease in children. We present interactive cases report where the different clinical forms of juvenile idiopathic arthritis are distinguished. We review the recent advances in diagnosis and treatment of interest for the primary care pediatrician.

Key words: Juvenile idiopathic arthritis. Diagnosis. Treatment.

\section{Introducción}

Las enfermedades reumáticas en la infancia y la adolescencia tienen gran relevancia en la actualidad, por ser consideradas una de las causas más importantes de reducción de la calidad de vi- da en el niño y el adolescente. Además, hay que considerar que la evolución crónica de la enfermedad no es tan favorable como inicialmente se creía, al comprobarse que, en la transición a la edad adulta, un porcentaje importante

Inmaculada Calvo Penadés: calvo_inm@gva.es

Los autores declaran no presentar conflictos de intereses en relación con la preparación y publicación de este artículo. 
de enfermos presentan actividad de enfermedad o discapacidad, ya sea debido a la persistencia de la inflamación a nivel articular o extraarticular de la enfermedad.

El mayor conocimiento del proceso inflamatorio de estas enfermedades en la última década ha permitido dar una nueva visión de la Reumatología Pediátrica: se han consensuado otros criterios de clasificación, se han creado nuevos instrumentos de medida de actividad de enfermedad y/o remisión y escalas de afectación orgánica, y se ha favorecido la aparición de nuevas herramientas para el diagnóstico de estas enfermedades, así como para el desarrollo de estrategias terapéuticas biológicas con el objetivo de conseguir la remisión de la enfermedad y evitar secuelas a corto y largo plazo.

Por otro lado, hay que reconocer dos grandes dificultades en el abordaje de esta patología: la gran complejidad para determinar y clasificar el tipo de enfermedad reumática, pues pueden pasar meses o años desde que se inician los síntomas hasta llegar al diagnóstico final; y su amplio contenido, dado que la Reumatología abarca en términos generales todas las alteraciones inflamatorias y no inflamatorias que afectan al tejido conectivo.
El objetivo de este seminario es estimular el interés del pediatra de Atención Primaria en el conocimiento de estas enfermedades, para poder participar tanto en la identificación precoz de estos pacientes como en su seguimiento clínico y terapéutico, estableciendo una coordinación asistencial con las Unidades de Reumatología Pediátrica. Para ello, trataremos el abordaje diagnóstico y terapéutico inicial a través de la resolución interactiva de unos casos clínicos representativos de las siguientes enfermedades:

- Artritis idiopática juvenil en sus distintas formas de expresión clínica; enfermedad considerada la más frecuente en esta especialidad, pues constituye entre un 50\% y un $60 \%$ de los casos con respecto al resto de enfermedades reumáticas. La variabilidad de la prevalencia recogida oscila entre 1 y 4 por cada 1000 niños, y la incidencia es de 10 a 22 casos nuevos al año, por cada 100000 niños. Se tratarán tanto el diagnóstico clínico inicial como su tratamiento y sus variaciones en función de la evolución clínica, así como las complicaciones más frecuentes y otros aspectos de interés en relación con su abordaje desde Atención Primaria, dado que es una 
enfermedad grave y crónica (vacunación, infecciones...).

- Enfermedades autoinflamatorias: estudio de la fiebre recurrente.

\section{Artritis idiopática juvenil}

\section{Aspectos relevantes \\ en el diagnóstico}

La última propuesta de clasificación disponible en este momento (Edmonton, 2001) tuvo el objetivo de unificar los criterios europeos y americanos. Así, se llegó al consenso para la denominación de la enfermedad como Artritis Idiopática Juvenil (AIJ) ${ }^{1-3}$ y se definieron los criterios para su diagnóstico, englobando todas las artritis que comenzaran antes de los 16 años de edad, que persistieran durante al menos seis semanas, al considerarse este periodo de tiempo suficiente para realizar un diagnóstico diferencial, que el diagnóstico precoz de la enfermedad permitiera un tratamiento temprano de la misma y, por último, la exclusión de otras causas conocidas de artritis. Este último criterio tiene una gran relevancia en la práctica clínica diaria, ya que no todo paciente juvenil con artritis puede diagnosticarse de AlJ (tabla 1).

Se considera que pueden identificarse siete formas clínicas de AIJ: sistémica (definida y probable), poliarticular factor reumatoide (FR) positivo, poliarticular FR negativo, oligoarticular persistente o extendida, artritis relacionada con entesitis, artritis psoriásica y otras artritis (actualmente indiferenciadas) (tabla 2).

Para establecer el diagnóstico de AlJ serán necesarias las seis semanas de evolución, y la clasificación de la forma clínica se realizará a los seis meses de evolución de la enfermedad, sobre la base de los criterios definidos anteriormente.

Los pacientes con AIJ sistémica constituyen aproximadamente un $10 \%$ de la totalidad de niños afectos de AIJ. Sin embargo, es en este grupo donde se encuentran los pacientes más graves, tanto por su afectación articular como por las complicaciones debidas a una actividad inflamatoria persistente (retraso del crecimiento, osteoporosis severa y amiloidosis, entre otras). La presencia de artritis es necesaria para poder realizar el diagnóstico de AlJ sistémica. La artritis puede afectar a una o más articulaciones a la vez, en las que comienzan los síntomas sistémicos, o bien seguir a estos en semanas o meses. Estos casos son los que presentan cierta dificultad diagnóstica, pues la fiebre puede estar presente mucho tiempo antes de que aparezca la primera manifestación 
articular. Aunque inicialmente se afecten pocas articulaciones, el curso tiende a ser con más frecuencia poliarticular.
La forma oligoarticular es la más frecuente dentro de la AlJ; afecta más a las niñas, con un pico de distribución entre

Tabla 1. Exclusiones en el diagnóstico de la artritis idiopática juvenil

\begin{tabular}{|c|}
\hline $\begin{array}{l}\text { Artritis infecciosa } \\
\text { - Bacterianas (tuberculosis, brucella) } \\
\text { - Virales } \\
\text { - Micóticas } \\
\text { - Enfermedad de Lyme }\end{array}$ \\
\hline $\begin{array}{l}\text { Artritis reactivas } \\
\text { - Salmonella } \\
\text { - Yersinia enterocolítica } \\
\text { - Chlamydia } \\
\text { - Campylobacter } \\
\text { - Fiebre reumática y artritis postestreptocócica }\end{array}$ \\
\hline $\begin{array}{l}\text { Enfermedades hematológicas } \\
\text { - Leucemia } \\
\text { - Linfoma } \\
\text { - Hemofilia } \\
\text { - Drepanocitosis }\end{array}$ \\
\hline $\begin{array}{l}\text { Enfermedades neoplásicas } \\
\text { - Histiocitosis } \\
\text { - Neuroblastoma }\end{array}$ \\
\hline $\begin{array}{l}\text { Enfermedades del tejido conectivo } \\
\text { - Lupus eritematoso sistémico } \\
\text { - Dermatomiositis } \\
\text { - Esclerodermia y síndromes asociados } \\
\text { - Enfermedad mixta del tejido conectivo } \\
\text { - Enfermedad de Sjögren } \\
\text { - PAN } \\
\text { - Enfermedad de Kawasaki } \\
\text { - Granulomatosis de Wegener } \\
\text { - Enfermedad Behçet }\end{array}$ \\
\hline $\begin{array}{l}\text { Trastornos osteoarticulares no inflamatorios } \\
\text { - Epifisiolosis } \\
\text { - Osteocondrosis } \\
\text { - Distrofia simpático refleja } \\
\text { - Traumatismos }\end{array}$ \\
\hline $\begin{array}{l}\text { Enfermedades metabólicas } \\
\text { - Mucopolisacaridosis } \\
\text { - Artropatía diabética }\end{array}$ \\
\hline $\begin{array}{l}\text { Otras } \\
\text { - Sarcoidosis } \\
\text { - Síndromes de fiebre periódica } \\
\text { - Enfermedad de Castelman } \\
\text { - Urticaria } \\
\text { - Vasculitis }\end{array}$ \\
\hline
\end{tabular}


Tabla 2. Criterios de clasificación de la artritis idiopática juvenil (ILAR, Edmonton 2001)

\begin{tabular}{|c|c|c|}
\hline Subtipo & Definición & Exclusiones \\
\hline Sistémica & $\begin{array}{l}\text { Artritis de una o más articulaciones con, o precedida de, fiebre diaria } \\
\text { de al menos dos semanas de duración, documentada al menos tres } \\
\text { días y acompañada de alguno de los siguientes elementos: } \\
\text { 1. Exantema evanescente, no fijo, eritematoso } \\
\text { 2. Adenomegalias generalizadas } \\
\text { 3. Hepatomegalia o esplenomegalias } \\
\text { 4. Serositis }\end{array}$ & $a, b, c, d$ \\
\hline Oligoarticular & $\begin{array}{l}\text { Artritis que afecta a 1-4 articulaciones durante los primeros seis } \\
\text { meses de la enfermedad. Dos subcategorías: } \\
\text { 1. Oligoarticular persistente } \\
\text { 2. Oligoarticular extendida }\end{array}$ & $a, b, c, d, e$ \\
\hline $\begin{array}{l}\text { Poliarticular } \\
\text { factor reumatoide } \\
\text { negativo }\end{array}$ & $\begin{array}{l}\text { Artritis que afecta a cinco o más articulaciones durante los } \\
\text { primeros seis meses de la enfermedad; test para FR negativo }\end{array}$ & $a, b, c, d, e$ \\
\hline $\begin{array}{l}\text { Poliarticular factor } \\
\text { reumatoide } \\
\text { positivo }\end{array}$ & $\begin{array}{l}\text { Artritis que afecta a cinco o más articulaciones durante los } \\
\text { primeros seis meses de la enfermedad, asociada con test para FR } \\
\text { positivos en dos ocasiones, separadas por tres meses }\end{array}$ & $a, b, c, e$ \\
\hline Psoriásica & $\begin{array}{l}\text { Artritis y psoriasis, o artritis y al menos dos de los siguientes: } \\
\text { 1. Dactilitis } \\
\text { 2. Punteado ungueal u onicolisis } \\
\text { 3. Historia familiar de psoriasis en al menos un pariente de primer } \\
\text { grado }\end{array}$ & $b, c, d, e$ \\
\hline $\begin{array}{l}\text { Artritis asociada } \\
\text { a entesitis }\end{array}$ & $\begin{array}{l}\text { Artritis y entesitis, o } \\
\text { Artritis o entesitis, con al menos dos de los siguientes: } \\
\text { 1. Presencia o historia de dolor a la palpación de la articulación } \\
\text { sacroiliaca y/o dolor espinal inflamatorio } \\
\text { 2. Presencia de HLA B } 27 \text { positivo } \\
\text { 3. Inicio de artritis en un varón mayor de seis años de edad } \\
\text { 4. Uveítis anterior aguda sintomática } \\
\text { 5. Historia de espondilitis anquilosante, artritis asociada a entesitis, } \\
\text { sacroileítis con enfermedad inflamatoria intestinal, síndrome de } \\
\text { Reiter, o uveítis anterior aguda en un familiar de primer grado }\end{array}$ & $a, d, e$ \\
\hline $\begin{array}{l}\text { Artritis } \\
\text { indiferenciada }\end{array}$ & $\begin{array}{l}\text { Niños con artritis de causa desconocida que persiste al menos } \\
\text { durante seis semanas, en los que: } \\
\text { 1. No se reúnen criterios diagnósticos para ninguna de las } \\
\text { categorías anteriores, o } \\
\text { 2. Se reúnen criterios diagnósticos para más de una categoría }\end{array}$ & \\
\hline
\end{tabular}

\section{Exclusiones}

a. Psoriasis o historia de psoriasis en el paciente o en un familiar de primer grado.

b. Artritis en un varón HLA B27 + que inicia después del sexto cumpleaños.

c. Espondilitis anquilosante, artritis asociada a entesitis, sacroileitis con enfermedad inflamatoria intestinal, síndrome de Reiter, o uveítis anterior aguda, o historia de uno de estos desórdenes en un familiar de primer grado. d.Presencia de factor reumatoide tipo IgM positivo en dos ocasiones separadas por al menos tres meses. e.Presencia de artritis idiopática juvenil sistémica en el paciente. 
uno y tres años, y se distinguen dos subcategorías:

- Oligoartritis persistente: afecta como máximo a cuatro articulaciones durante toda la evolución de la enfermedad.

- Oligoartritis extendida: afecta a un total de cinco o más articulaciones tras los seis meses de evolución de la enfermedad.

Hay que tener en cuenta las exclusiones (ver tabla 2) y el riesgo de uveítis anterior o iridociclitis. Esta última está considerada como la manifestación extraarticular más importante de la AlJ oligoarticular. Se presenta con una frecuencia aproximada entre un $15 \%$ y un $20 \%$ respecto al $5 \%$ en la forma poliarticular. Habitualmente, la artritis precede al desarrollo de la uveítis, que suele aparecer entre los los dos y los cinco años siguientes al inicio de la enfermedad articular, aunque por lo general el máximo riesgo de aparición de uveítis tiene lugar en los dos años siguientes a la aparición de la artritis. En la mayoría de los pacientes, la inflamación ocular es sintómatica, pero en ocasiones se puede presentar sin sintomatología acompañante, lo que sugiere la necesidad de la exploración del oftalmólogo con lámpara de hendidura y la aplicación normalizada de un protocolo en el seguimiento clínico ${ }^{4}$.
La forma poliarticular representa aproximadamente el $30 \%$ de todas las AIJ, correspondiendo el $10 \%$ a la forma $\mathrm{FR}+\mathrm{y}$ el $20 \%$ a la forma FR-, que tiene mejor pronóstico. La enfermedad afecta más a las niñas (relación 3:1). La edad de comienzo sigue un patrón bifásico, con un pico temprano entre el primer y el cuarto año de vida y otro pico tardío, que comienza alrededor de los 14 años, considerado como el equivalente en la infancia de la artritis reumatoide del adulto. El FR es el principal marcador inmunogenético de esta enfermedad. La presencia de anticuerpos antinucleares (ANA) positivos, se observa en el 40-50\% de los pacientes. Son características de las formas iniciales de FR+: la presencia de sinovitis dorsal de los carpos, junto con sinovitis de los extensores de las manos; la artritis de cadera, si se presenta precozmente como marcador pronóstico; y la afectación precoz de la columna cervical y de las temporomandibulares como signo de gravedad.

La forma más frecuente de presentación de la artritis psoriásica es en forma de una artritis oligoarticular ${ }^{5}$ asimétrica que afecta a grandes y pequeñas articulaciones, acompañada frecuentemente de dactilitis, con evolución poliarticular, riesgo de padecer uveitís crónica, ANA 
positivos, FR negativo y HLA-B27 negativo, con pico máximo entre los ocho y los diez años. Generalmente, estos pacientes tienen la artritis como primera manifestación y después la psoriasis (40-50\%), en muy pocos casos la presentación es simultánea (10\%). La aparición de la psoriasis ocurre con más frecuencia, alrededor de los dos años de evolución de la artritis, aunque en ocasiones el intervalo puede ser mayor de 14 años ${ }^{5}$.

En la forma artritis entesitis, la entesitis junto y la presencia de artralgia/artritis constituyen dos signos guía que, junto con la negatividad de los marcadores biológicos de otras formas de AIJ (FR y ANA), permiten identificar con bastante aproximación a estos pacientes sin necesidad de recurrir al HLA-B27 ${ }^{6}$. La asociación entre HLA-B27 y la espondiloartritis juvenil, definida según los criterios de Nueva York o del Grupo Europeo para el estudio de Espondiloartritis (ESSG), es tan marcada como en el adulto?. La forma más habitual de inicio es una oligoartritis asimétrica. La presencia de tarsitis, es muy característica y produce una discapacidad moderada. Las manifestaciones axiales con dolor en sacroiliacas pueden tardar años en presentarse, el dolor cervical, con moderada limitación de la movilidad puede presentarse desde los primeros síntomas de la enfermedad ${ }^{8}$. La localización aquilea es la entesitis más frecuente aunque también la fascia plantar, pudiendo ser la primera manifestación de la enfermedad.

\section{Avances en el tratamiento}

El tratamiento adecuado de este grupo de enfermedades comienza con la identificación precoz de la enfermedad. El objetivo principal es conseguir frenar la actividad y evitar secuelas importantes.

Para ello, no hay que olvidar que, además del tratamiento farmacológico, hay otros aspectos importantes que deben considerarse, como son la fisioterapia, que ayuda a reducir el dolor y mantener la fuerza muscular; la valoración sistemática del crecimiento; las inmunizaciones reglamentarias, no administrando vacunas de virus vivos si el paciente recibe tratamiento con corticoides en dosis altas o inmunodepresores; el tratamiento adecuado de las infecciones; y el tratamiento en la alteración de la densidad mineral ósea que muestran los niños con enfermedades reumáticas.

La utilización de antiinflamatorios no esteroideos (AINE) como tratamiento coadyuvante para el dolor y el bienestar del paciente sigue estando indicado, al 
igual que el uso de corticoides en dosis bajas como tratamiento de rescate, 0 bien solo de forma excepcional, en la AIJ sistémica o en enfermedades autoinmunitarias (lupus eritematoso sistémico y dermatomiositis juvenil); dosis altas orales diarias; o bien tratamiento en pulsos (altas dosis intravenosas), para poder frenar los brotes de la enfer- medad o complicaciones graves del paciente.

El tratamiento farmacológico se individualizará en función de las manifestaciones clínicas y el grado de afectación orgánica, además de intentar conseguir una integración social completa tanto en la escolarización como en la actividad física, lo más parecida a un

Tabla 3. Fármacos inductores de remisión o inmunomoduladores

\begin{tabular}{c|c|c|c}
\hline Tipo & Dosis & Indicación & Toxicidad \\
\hline Metotrexato & $0,3-0,5 \mathrm{mg} / \mathrm{kg} /$ semana & $\begin{array}{c}\text { Artritis idiopática juvenil } \\
\text { Enfermedades autoinmunitarias } \\
\text { Vasculitis }\end{array}$ & $\begin{array}{c}\text { Náuseas } \\
\text { Mucositis } \\
\text { Hepatopatía }\end{array}$ \\
\hline Leflunamida & $10 \mathrm{mg} / \mathrm{kg} /$ día $<40 \mathrm{~kg}$ & Artritis idiopática juvenil & Cefalea \\
& $20 \mathrm{mg} / \mathrm{kg} /$ día $>40 \mathrm{~kg}$ & & \\
\hline
\end{tabular}

Tabla 4. Fármacos inmunodepresores

\begin{tabular}{|c|c|c|c|}
\hline Tipo & Dosis & Indicación & Toxicidad \\
\hline Ciclosporina & 3-5 mg/kg/día & $\begin{array}{c}\text { AlJ sistémica } \\
\text { LES }\end{array}$ & $\begin{array}{c}\text { Hirsutismo } \\
\text { Nefropatía } \\
\text { HTA }\end{array}$ \\
\hline Tacrolimus & 0,07-1 mg/kg/día & $\begin{array}{c}\text { AlJ sistémica } \\
\text { DMJ }\end{array}$ & $\begin{array}{l}\text { Infecciones } \\
\text { Afectación } \\
\text { neurológica }\end{array}$ \\
\hline Azatioprina & $1,5-3 \mathrm{mg} / \mathrm{kg} / \mathrm{día}$ & $\begin{array}{c}\text { AlJ } \\
\text { Enfermedades } \\
\text { autoinmunitarias } \\
\text { Vasculitis }\end{array}$ & $\begin{array}{c}\text { Mielodepresión } \\
\text { Hepatopatía }\end{array}$ \\
\hline Ciclofosfamida & $400 \mathrm{mg} / \mathrm{m}^{2} / \mathrm{mes}$ & $\begin{array}{c}\text { LES } \\
\text { Vasculitis }\end{array}$ & $\begin{array}{c}\text { Mielodepresión } \\
\text { Cistitis } \\
\text { Neoplasias }\end{array}$ \\
\hline $\begin{array}{l}\text { Micofenolato } \\
\text { mofetil }\end{array}$ & 300 mg/m²/día & $\begin{array}{l}\text { AlJ sistémica } \\
\text { LES }\end{array}$ & Gastrointestinales \\
\hline Gammaglobulina & $1,5 \mathrm{~g} / 15 \mathrm{~d} / 2 \mathrm{~m}$ & $\begin{array}{l}\text { AlJ sistémica } \\
\text { DMJ }\end{array}$ & $\begin{array}{l}\text { Meningitis } \\
\text { aséptica }\end{array}$ \\
\hline
\end{tabular}


niño normal. Si tenemos en cuenta este planteamiento, entenderemos por qué, en la actualidad, muchos de los reumatólogos pediátricos introducen de forma precoz fármacos modificadores de la enfermedad, inductores de remisión o inmunomoduladores, y no se limitan solo a instaurar un tratamiento antiinflamatorio convencional durante los primeros meses de la enfermedad (tablas 3 y 4). Así, la pirámide terapéutica en este grupo de enfermedades ha cambiado considerablemente en estos últimos diez años. Considerando además el nacimiento de los agentes biológicos y la aplicación del trasplante de células madre en las enfermedades autoinmunitarias (tabla 5), se ha conseguido en gran medida la mejoría del pronóstico de estas enfermedades ${ }^{9,10}$.

\section{Conclusiones}

Para poder diagnosticar las enfermedades reumáticas es fundamental, en primer lugar, conocerlas, lo que a su vez permite reconocerlas a partir de una serie de signos y síntomas variados que presentan estos niños y que motivan, en muchas ocasiones, su consulta en diversos servicios médicos antes de llegar a Reumatología (Pediatría de Atención Primaria, Traumatología, Dermatología, Urgencias...). En este sentido, el Pediatra de Atención Primaria es el facultativo que con mayor precocidad puede

\begin{tabular}{l|c|c|c}
\hline \multicolumn{1}{c|}{ Tabla 5. Fármacos biológicos } & \multicolumn{3}{c}{ Toxicidad } \\
\hline \multicolumn{1}{c|}{ Tipo } & Dosis & Indicación & $\begin{array}{c}\text { Infecciones } \\
\text { recurrentes }\end{array}$ \\
\hline Etanercept & $0,4 \mathrm{mg} / \mathrm{kg} / \mathrm{semana}$ SC & AlJ poliarticular & $\begin{array}{c}\text { Anafilaxia } \\
\text { TBC }\end{array}$ \\
\hline Infliximab & $3-5 \mathrm{mg} / \mathrm{kg} / 2$ meses IV & $\begin{array}{c}\text { AlJ poliarticular } \\
\text { Vasculitis } \\
\text { Enfermedad de Cronh }\end{array}$ & Reacción local \\
\hline Adalimumab & $24 \mathrm{mg} / \mathrm{m}^{2} / 15$ días IV & $\begin{array}{c}\text { AlJ } \\
\text { Uveítis crónica } \\
\text { Enfermedad de Cronh }\end{array}$ & \\
\hline Anakinra & $1-5 \mathrm{mg} / \mathrm{kg} /$ día SC & AlJ sistémica & Infecciones \\
\hline Tocilizumab & $8-12 \mathrm{mg} / \mathrm{kg} / 15$ días & AlJ sistémica & Infecciones \\
\hline Abatacept & $10 \mathrm{mg} / \mathrm{kg} / \mathrm{mes}$ & AlJ poliarticular & Infecciones \\
\hline Rituximab & $300 \mathrm{mg} / \mathrm{m}^{2} / \mathrm{semanal}$ & AlJ sistémica & Infecciones \\
& & LES & \\
\hline
\end{tabular}

AlJ: artritis idiopática juvenil; IV: vía intravenosa; LES: lupus eritematoso sistémico; SC: vía subcutánea; TBC: tuberculosis. 
detectar la patología reumática y, por lo tanto, influir en su pronóstico. La actualización de los conocimientos en esta patología ayuda a identificar más precozmente estas enfermedades y mejora su seguimiento en Atención Primaria en coordinación con las Unidades de Reumatología.
Para concluir, esperamos que el diagnóstico precoz de estas enfermedades, junto con la aplicación más precoz de tratamientos más individualizados, y un mayor conocimiento de las nuevas terapias biológicas cambien en un futuro muy corto los resultados pronósticos de actividad de estas entidades.

\section{Bibliografía}

1. Fink CW. Proposal for the development of classification criteria for idiopathic arthritis of childhood. J Rheumatol. 1995;22:1566-9.

2. Petty RE, Southwood TR, Baum J, Bhettay E, Glass DN, Manners P et al. Revision of the proposed classification criteria for juvenile idiopathic arthritis. Durban 1997. J Rheumatol. 1998;25:1991-4.

3. Petty RE, Southwood TR, Manners P. International League of Associations for rheumatology classification of juvenile idiopathic arthritis: second revision. Edmonton 2001. J Rheumatol. 2004;31:390-2.

4. American Academy of Pediatrics Section on Rheumatology ans Section on Ophtalmology. Guidelines for ophthalmologic examination in children with juvenile rheumatoid arthritis. Pediatrics. 1993;92:295-6.
5. Southwood TR. Psoriasic Arthritis. En: Textbook of Paediatric Rheumatology. Philadelphia: Saunders Company; 2001. p. 345-55.

6. Rosenberg A, Petty RE. A syndrome of seronegative enthesopaty and arthrotphaty children. Arthritis Rheumatol. 1982;25:1041-9.

7. Burgos-Vargas R, Vazquez Mellado J. The early clinical recognition of juvenile-onset ankylosing spondylitis and its differentiation from juvenile rheumatoid arthritis. Arthritis Rheumatol. 1995;38:835-44.

8. Burgos-Vargas R, Pacheco Tena C, Vazquez Mellado J. Juvenile-onset spondyloarthropathies. Rheumatol Clin Nort Am. 1997;23:569-849.

9. Calvo I. Tratamiento médico de la artritis idiopática Juvenil. Capítulo 6. Monografía SER Reumatología Pediátrica. Editorial Médica Panamericana; 2007. p. 97-112.

10. Cimaz R. Actualización en Reumatología Pediátrica. An Pediatr (Barc). 2005;63:293-8. 